\title{
Association of genetic polymorphism of HLA-DRB1 antigens with the susceptibility to lepromatous leprosy
}

\author{
MONICA ESCAMILLA-TILCH ${ }^{1}$, NORA MAGDALENA TORRES-CARRILLO ${ }^{2}$, ROSALIO RAMOS PAYAN $^{3}$, \\ MARIBEL AGUILAR-MEDINA ${ }^{3}$, MA ISABEL SALAZAR ${ }^{1}$, MARY FAFUTIS-MORRIS ${ }^{4,5}$, \\ ROBERTO ARENAS-GUZMAN ${ }^{6}$, SERGIO ESTRADA-PARRA ${ }^{1}$, IRIS ESTRADA-GARCIA ${ }^{1}$ and JULIO GRANADOS ${ }^{7}$ \\ ${ }^{1}$ Department of Immunology, National School of Biological Sciences, National Polytechnic Institute, Mexico City 11340; \\ ${ }^{2}$ Department of Basic Science Research, National Institute of Geriatrics, Ministry of Health, Mexico City, 10200; ${ }^{3}$ School \\ of Chemical and Biological Sciences, Autonomous University of Sinaloa, Culiacan, Sinaloa 80040; ${ }^{4}$ Center for Research in \\ Immunology and Dermatology, Dermatological Institute of Jalisco, Guadalajara, Jalisco 45190; \\ ${ }^{5}$ Department of Physiology, University Center of Health Sciences, University of Guadalajara, Guadalajara, Jalisco 44100; \\ ${ }^{6}$ Mycology Section, General Hospital Dr Manuel Gea Gonzalez; ${ }^{7}$ Department of Transplantation, National \\ Institute of Medical Sciences and Nutrition Salvador Zubiran, Mexico City 14000, Mexico
}

Received August 07, 2013; Accepted September 16, 2013

DOI: $10.3892 /$ br.2013.167

\begin{abstract}
Despite the introduction of multidrug therapy and the overall reduction of leprosy prevalence in Mexico, the disease remains endemic in certain regions of the country. A genetic basis for the immune susceptibility to Mycobacterium leprae has already been established in different populations worldwide. In this study, we investigated the possible association of the HLA-DRB1 alleles with leprosy in a Mexican Mestizo population. The results demonstrated that the HLA-DRB1*01 allele is associated with lepromatous and dimorphic leprosy $[\mathrm{P}<0.001$, odds ratio $(\mathrm{OR})=4.6,95 \%$ confidence interval $(95 \% \mathrm{CI}): 1.8-11.4$; and $\mathrm{P}=0.03, \mathrm{OR}=6.2,95 \% \mathrm{CI}$ : 1.1-31.6, respectively] and the frequency of the HLA-DRB $1 * 08$ allele was found to be significantly lower among leprosy patients compared to controls $(\mathrm{P}=0.046, \mathrm{OR}=2.4,95 \% \mathrm{CI}: 1-5.8)$. In conclusion, although the association of the HLA-DR locus with leprosy has been established in different populations and several studies have demonstrated significant differences in the DR alleles, this study demonstrated an association of the HLA-DRB1*01 allele with susceptibility to lepromatous and dimorphic leprosy, as
\end{abstract}

Correspondence to: Dr Julio Granados, Department of Transplantation, National Institute of Medical Sciences and Nutrition Salvador Zubiran, 15 Vasco de Quiroga, Colonia Seccion XVI, Delegacion Tlalpan, Mexico City 14000, Mexico

E-mail: julgrate@yahoo.com

Dr Iris Estrada-Garcia, Department of Immunology, National School of Biological Sciences, National Polytechnic Institute, Prolongacion de Carpio y Plan de Ayala s/n, Colonia Santo Tomas, Mexico City 11340, Mexico

E-mail: iestrada5@hotmail.com

Key words: leprosy, HLA-DRB1, polymorphism well as an association of the HLA-DRB1*08 allele with protection against leprosy in a Mexican Mestizo population.

\section{Introduction}

Leprosy is a chronic infectious disease caused by the intracellular acid-fast Mycobacterium leprae (M. leprae) bacilli. It affects the skin and peripheral nerves of susceptible individuals, causing irreversible impairment of nerve function and consequent chronic disability (1). As a result of the use of multidrug therapy, promoted by the World Health Organization, the global prevalence of this disease has been significantly reduced $(2,3)$. However, leprosy remains a public health problem (4), affecting 500,000 individuals annually worldwide (3). In Mexico, 215 new cases of leprosy were reported until the end of the first quarter of 2012, resulting in an estimated prevalence of 0.480 cases per 100,000 inhabitants, with a predominance of males over females. In addition, the proportion of multibacillary cases has been found to increase in several Mexican populations, concomitantly with the declining incidence (2,5). However, a previous epidemiological study demonstrated that only a small percentage of individuals exposed to the bacillus develop the disease, suggesting that the majority of individuals are immunologically competent to reject infection by developing effective cellular immune responses (6).

The clinical spectrum of leprosy includes two poles, the tuberculoid (TT) or paucibacillary (PB) and the lepromatous (LL) or multibacillary (MB) poles, with several intermediate (I) or borderline forms [borderline tuberculoid (BT), borderline borderline (BB) and borderline lepromatous (BL)]. Immunologically, the LL pole is characterized by a Th2 T-cell immune response, antibody complex formation, absence of granulomas and failure to restrain M. leprae growth. By contrast, the TT pole presents with a Th1 T-cell cytokine response, a vigorous $\mathrm{T}$-cell response 
to $M$. leprae antigens and containment of the infection in well-formed granulomas (7).

Accumulated evidence currently indicates that exposure to $M$. leprae is necessary but not sufficient to explain the susceptibility to leprosy and previous studies demonstrated that the pathogenesis of leprosy is a two-step process, in which a group of genes controls susceptibility to infection per se, whereas a different group of genes controls the clinical manifestation of the disease $(1,8)$. Evidence that host genes affect susceptibility to leprosy or its various clinical forms is supported by data from a wide variety of sources, including twin studies, segregation analyses, family-based linkage and association studies, candidate gene association studies and, most recently, genome-wide association studies (GWASs) $(7,9)$. One recent genome-wide scan pinpointed chromosome $6 \mathrm{p} 21$ as a leprosy susceptibility locus (10). This region harbors the human leukocyte antigens (HLA) gene cluster, which has been extensively investigated for its role in the pathogenesis of leprosy. Previous candidate gene studies detected an association of leprosy with class I and II genes. However, class II genes, particularly the DRB1 locus, were found to be more consistently associated with leprosy $(6,11)$.

Several studies reported an association of the HLA-DR locus with susceptibility or resistance to leprosy in different populations, demonstrating the significance of this locus in the pathogenesis of leprosy and highlighting the fact that certain HLA-DR alleles are associated with susceptibility to leprosy per se, whereas other alleles are associated with predisposition towards a particular clinical spectrum of this disease (12). In this context, independent replication studies in several ethnically different populations are required for multifactorial diseases, such as leprosy (6). Considering these facts and taking into consideration that the genetic susceptibility to leprosy in the Mexican Mestizo population has received little attention, the aim of this study was to investigate the association of HLA-DRB1 alleles with leprosy in a Mexican Mestizo population.

\section{Subjects and methods}

Subjects. In this study, we recruited a total of 52 patients with leprosy ( $39 \%$ female and $61 \%$ male), with a mean age of $65 \pm 14$ years (range, $18-86$ years), who were classified according to the international criteria establish by Ridley and Jopling (13). The patients were residents from the states of Sinaloa (48\%), Jalisco (27\%), Guanajuato (11\%), Oaxaca (6\%), Veracruz (2\%), Nuevo Leon (2\%), Guerrero (2\%) and Hidalgo (2\%). Forty-one patients were classified as LL, 2 as TT, 6 as dimorphic (D) and 3 as I. All leprosy cases were MB, with the exception of 5 PB (i.e., 2 TT and 3 I). A total of 99 healthy individuals (50\% female and $50 \%$ male), with a mean age of $40 \pm 10$ years (range, 28-52 years), unrelated to the patients and matched by ethnicity, were included as the control group. Ethnically, the patients and the controls were classified as Mestizos, who are defined as individuals born in Mexico, with a Spanish-derived last name and Mexican ancestors at least back to the third generation. Mestizos are the result of 500 years of admixture between Spaniards, Amerindians and Africans and they currently represent the majority of the Mexican population (>90\%) $(14,15)$.
A written informed consent was obtained from all the subjects prior to their enrollment in this study, according to the Helsinki Declaration.

HLA-DRB1 typing. Genomic DNA from all the subjects included in this study was purified from peripheral blood leukocytes, according to the method described by Miller (16). Blood was collected by single peripheral venepuncture, according to guidelines approved by the Internal Review Boards of the participant hospitals. The HLA-DRB1 locus was genotyped based on the hybridization of labeled single-stranded polymerase chain reaction products to sequence-specific oligonucleotides, using the LifeCodes HLA-DRB1 Typing kit for use with Luminex (Gen-Probe Transplant Diagnostics, Inc., Stamford, CT, USA) following the manufacturer's recommendations. Data were analyzed using Quicktype for Lifecodes version 3.0 software to determine the HLA alleles.

Statistical analysis. The allele frequencies were calculated by direct counting and the differences in the distribution of the alleles between patients and controls were analyzed using the $\chi^{2}$ test or the Fisher's exact test. Statistically significant P-values $(\leq 0.05)$ were corrected taking into consideration the number of alleles observed (pc) (17). The strength of the associations was estimated by calculating the odds ratio (OR). Statistical analyses were performed using Arlequin software, version 3.5.1.2 (Swiss National Science Foundation, Bern, Switzerland).

\section{Results}

Subjects and allele frequency. A total of 151 Mexican Mestizo individuals (52 patients with leprosy and 99 healthy controls) were genotyped for the HLA-DRB1 locus. Table I summarizes the allelic frequency distributions of the HLA-DRB1 alleles in leprosy patients and controls. Of the 13 HLA-DRB1 alleles determined $(* 01, * 03, * 04, * 07$, $* 08, * 11, * 13, * 14$ and $* 15$ being the more frequent) we only identified 2 alleles exhibiting a statistically significant difference in frequency between patients and controls. The frequency of the HLA-DRB1*01 allele among leprosy patients was significantly higher compared to that observed among healthy controls $[\mathrm{P}<0.001, \mathrm{OR}=5.6,95 \%$ confidence interval (95\% CI): 2.4-13.3]. By contrast, the frequency of the HLA-DRB1*08 allele was significantly lower among leprosy patients compared to that among controls $(\mathrm{P}=0.046, \mathrm{OR}=2.4$, 95\% CI: 1-5.8) (Table I).

Association of alleles with leprosy subtypes. We investigated the possible association of the HLA-DRB1 alleles with the clinical subtypes of leprosy. For this purpose, taking into consideration that the majority of cases of leprosy in Mexico are $\mathrm{MB}$ and due to the fact that only 5 samples were collected from patients with the $\mathrm{PB}$ form, we only considered the MB form and healthy controls for this analysis. We observed that the frequency of the HLA-DRB1*01 allele was significantly higher in the MB group compared to that among controls $(\mathrm{P}<0.001$, $\mathrm{OR}=4.5$, 95\% CI: 1.84-11.4) (Table II). This association was confirmed when we performed the analysis in the groups 
Table I. Frequencies of HLA-DRB1 alleles in patients with leprosy and healthy controls.

\begin{tabular}{|c|c|c|c|c|c|c|c|}
\hline \multirow{2}{*}{$\begin{array}{l}\text { HLA-DR } \\
\text { alleles }\end{array}$} & \multicolumn{2}{|c|}{ Leprosy $(\mathrm{N}=52)$} & \multicolumn{2}{|c|}{ Controls $(\mathrm{N}=99)$} & \multirow[b]{2}{*}{$\mathrm{pc}$} & \multirow[b]{2}{*}{ OR } & \multirow[b]{2}{*}{$95 \% \mathrm{CI}$} \\
\hline & $\mathrm{n}$ & $\mathrm{gf}$ & $\mathrm{n}$ & $\mathrm{gf}$ & & & \\
\hline 01 & 24 & 0.230 & 10 & 0.050 & $<0.001$ & 5.6 & 2.4-13.3 \\
\hline 04 & 20 & 0.192 & 47 & 0.237 & 0.45 & - & - \\
\hline 07 & 18 & 0.173 & 22 & 0.111 & 0.18 & - & - \\
\hline 08 & 8 & 0.077 & 33 & 0.167 & $0.046^{\mathrm{a}}$ & 2.4 & $1-5.8$ \\
\hline 13 & 8 & 0.077 & 10 & 0.050 & 0.50 & - & - \\
\hline 14 & 6 & 0.057 & 21 & 0.106 & 0.23 & - & - \\
\hline 15 & 6 & 0.057 & 13 & 0.065 & 0.98 & - & - \\
\hline 03 & 6 & 0.057 & 11 & 0.055 & 0.93 & - & - \\
\hline 11 & 4 & 0.040 & 20 & 0.101 & 0.092 & - & - \\
\hline 10 & 2 & 0.019 & 1 & 0.005 & 0.273 & - & - \\
\hline 09 & 1 & 0.009 & 3 & 0.015 & 1 & - & - \\
\hline 12 & 0 & 0 & 2 & 0.010 & 0.546 & - & - \\
\hline 16 & 1 & 0.009 & 5 & 0.025 & 0.668 & - & - \\
\hline
\end{tabular}

$\mathrm{N}$, number of individuals; gf, genetic frequencies; n, number of alleles; pc, Yates corrected P-values corrected taking into consideration the number of alleles observed; OR, odds ratio; 95\% CI, 95\% confidence interval. anverted value of OR.

Table II. Frequencies of HLA-DRB1 alleles in multibacillary (MB) patients and healthy controls.

\begin{tabular}{|c|c|c|c|c|c|c|c|}
\hline \multirow{2}{*}{$\begin{array}{l}\text { HLA-DR } \\
\text { alleles }\end{array}$} & \multicolumn{2}{|c|}{$\mathrm{MB}(\mathrm{N}=41)$} & \multicolumn{2}{|c|}{ Controls $(\mathrm{N}=99)$} & \multicolumn{3}{|c|}{ MB vs. controls } \\
\hline & $\mathrm{n}$ & gf & $\mathrm{n}$ & gf & $\mathrm{pc}$ & OR & $95 \% \mathrm{CI}$ \\
\hline 01 & 16 & 0.195 & 10 & 0.050 & $<0.001$ & 4.5 & $1.84-11.4$ \\
\hline 04 & 16 & 0.195 & 47 & 0.237 & 0.53 & - & - \\
\hline 07 & 14 & 0.171 & 22 & 0.111 & 0.24 & - & - \\
\hline 08 & 8 & 0.098 & 33 & 0.166 & 0.19 & - & - \\
\hline 13 & 7 & 0.085 & 10 & 0.050 & 0.40 & - & - \\
\hline 14 & 5 & 0.061 & 21 & 0.106 & 0.33 & - & - \\
\hline 15 & 4 & 0.049 & 13 & 0.065 & 0.79 & - & - \\
\hline 11 & 4 & 0.049 & 20 & 0.101 & 0.23 & - & - \\
\hline 03 & 3 & 0.037 & 11 & 0.055 & 0.71 & - & - \\
\hline 09 & 1 & 0.012 & 3 & 0.015 & 0.71 & - & - \\
\hline 10 & 2 & 0.02 & 1 & 0.005 & 0.42 & - & - \\
\hline 16 & 1 & 0.012 & 5 & 0.025 & 0.81 & - & - \\
\hline 12 & 1 & 0.012 & 2 & 0.010 & 0.89 & - & - \\
\hline
\end{tabular}

$\mathrm{N}$, number of individuals; $\mathrm{n}$, number of alleles; gf, genetic frequencies; pc, Yates corrected P-values taking into consideration the number of alleles observed; OR, odds ratio; 95\% CI, 95\% confidence interval.

based on clinical classification, where we observed a strong association of the HLA-DRB1*01 allele with LL and D leprosy $(\mathrm{P}<0.001, \mathrm{OR}=4.6,95 \% \mathrm{CI}: 1.8-11.4$; and $\mathrm{P}=0.03, \mathrm{OR}=6.2$, 95\% CI: 1.1-31.6, respectively) (Table III). Furthermore, as regards the HLA-DRB1*07 allele, we observed that there was a statistical tendency to $\mathrm{D}$ leprosy, although the association was not statistically significant $(\mathrm{P}=0.069)$. In the case of the other HLA-DRB1 alleles, no statistically significant differences were observed between patients and controls $(\mathrm{P} \geq 0.05)$ (Table III).

\section{Discussion}

It has been established that certain individuals who are exposed to the $M$. leprae bacillus are resistant to disease and raise an effective immune response, whereas others will develop clinical disease (18), suggesting that the development of leprosy is significantly affected by host genetics factors. An observation supporting the significant role of genetic factors in the development of leprosy is that several genes and genomic regions have been implicated in the 
Table III. Frequencies of HLA-DRB1 alleles in leprosy subgroups and healthy controls.

\begin{tabular}{|c|c|c|c|c|c|c|c|c|c|c|c|c|}
\hline \multirow{2}{*}{$\begin{array}{l}\text { HLA-DR } \\
\text { alleles }\end{array}$} & \multicolumn{2}{|c|}{ LL (N=41) } & \multirow[b]{2}{*}{$\mathrm{pc}^{\mathrm{a}}$} & \multirow[b]{2}{*}{ OR } & \multirow[b]{2}{*}{$95 \% \mathrm{CI}$} & \multicolumn{2}{|c|}{$\mathrm{D}(\mathrm{N}=6)$} & \multirow[b]{2}{*}{$\mathrm{pc}^{\mathrm{b}}$} & \multirow[b]{2}{*}{ OR } & \multirow[b]{2}{*}{$95 \%$ CI } & \multicolumn{2}{|c|}{$\begin{array}{l}\text { Controls } \\
(\mathrm{N}=99)\end{array}$} \\
\hline & $\mathrm{n}$ & $\mathrm{gf}$ & & & & $\mathrm{n}$ & gf & & & & $\mathrm{n}$ & gf \\
\hline 01 & 16 & 0.195 & $<0.001$ & 4.6 & $1.8-11.4$ & 3 & 0.250 & 0.03 & 6.2 & $1.1-31.6$ & 10 & 0.050 \\
\hline 03 & 5 & 0.061 & 0.91 & - & - & 0 & 0 & 0.86 & - & - & 11 & 0.055 \\
\hline 04 & 16 & 0.195 & 0.53 & - & - & 2 & 0.167 & 0.83 & - & - & 47 & 0.237 \\
\hline 07 & 14 & 0.171 & 0.24 & - & - & 4 & 0.333 & 0.069 & 4 & $0.9-16.2$ & 22 & 0.111 \\
\hline 09 & 1 & 0.012 & 0.31 & - & - & 0 & 0 & 0.41 & - & - & 3 & 0.015 \\
\hline 10 & 2 & 0.02 & 0.42 & - & - & 0 & 0 & 0.05 & - & - & 1 & 0.005 \\
\hline 12 & 0 & 0 & 0.89 & - & - & 0 & 0 & 0.23 & - & - & 2 & 0.010 \\
\hline 08 & 8 & 0.098 & 0.19 & - & - & 0 & 0 & 0.25 & - & - & 33 & 0.167 \\
\hline 13 & 6 & 0.073 & 0.64 & - & - & 2 & 0.167 & 0.29 & - & - & 10 & 0.050 \\
\hline 14 & 5 & 0.061 & 0.33 & - & - & 1 & 0.083 & 0.81 & - & - & 21 & 0.106 \\
\hline 15 & 4 & 0.049 & 0.79 & - & - & 0 & 0 & 0.76 & - & - & 13 & 0.065 \\
\hline 11 & 4 & 0.049 & 0.23 & - & - & 0 & 0 & 0.51 & - & - & 20 & 0.101 \\
\hline 16 & 1 & 0.012 & 0.75 & - & - & 0 & 0 & 0.67 & - & - & 5 & 0.025 \\
\hline
\end{tabular}

LL, lepromatous leprosy; N, number of individuals; D, dimorphic leprosy; n, number of alleles; gf, genetic frequencies; pc, Yates corrected P-values taking into consideration the number of alleles observed; OR, odds ratio; 95\% CI, 95\% confidence intervals. ${ }^{a} \mathrm{LL}$ vs. controls; ${ }^{\mathrm{b}} \mathrm{D}$ vs. controls.

complex genetic mechanisms controlling host susceptibility to disease (1).

Leprosy is one of the first human diseases in which human leukocyte antigen genes were demonstrated to co-determine disease outcome (18). These associations with HLA molecules may be the major genetic determinants of the disease phenotype, which is consistent with the large numbers of available studies that demonstrated an association between the DR alleles and the different clinical subtypes of leprosy, or even with leprosy per se (19).

It was previously demonstrated that, in Mexicans, the HLA-DRB $1 * 03$ allele is associated with the TT clinical subgroup (20) and a previous family study demonstrated an association of the HLA-DRB1*1501 allele with LL leprosy (21). In this study, we reported the association of the HLA-DRB1*01 allele with susceptibility to LL and D leprosy and we observed that the HLA-DRB1*08 allele was associated with protection against leprosy. Of note, the HLA-DRB1*01 allele appears to have been introduced in this population through admixture with Caucasians, whereas the HLA-DRB1*08 allele is the second most common allele encountered in the normal Mexican population and it is present in Mestizos, as well as in autochthonous populations from Mexico, which may be associated with natural selection from infectious diseases following contact with Europeans during the 16th century (22). These data confirm the role of HLA in the genetic susceptibility to the development of leprosy in several populations worldwide and also confirm the role of HLA-DR in the clinical outcome of this disease.

As regards other endemic countries, such as Vietnam and Brazil, the HLA-DRB1*10 allele has been associated with susceptibility to leprosy, whereas the HLA-DRB1*04 allele has been associated with protection against leprosy (6). A previous study from Brazil demonstrated that the HLA-DRB1*09 allele was associated with susceptibility to and the HLA-DRB1*04 allele with protection against BL (23). In Argentina, the HLADRB1*04 allele was found to be associated with protection against MB and the HLA-DRB1*0402 allele with protection against leprosy per se (24).

In a Chinese population, the HLA-DRB1*09 allele was associated with the development of indeterminate leprosy only in young people, whereas the HLA-DRB1*15 allele was associated with susceptibility to leprosy per se (12). The HLA-DRB1*1501, HLA-DRB1*1502 and HLADR-B1*1404 alleles were associated with an increased susceptibility to TT in an Indian population (25). A study from Indonesia demonstrated that the HLA-DRB1*02 allele confers susceptibility to, whereas the HLA-DRB1*12 allele is associated with protection against leprosy (26). In Egypt, HLA-DR2 was associated with susceptibility to leprosy per se (27) and in northern India, the HLA-DRB1*15 allele (a HLA-DR2 subtype) was associated with MB (28).

Considering those results, to the best of our knowledge, this study was the first to report evidence of an association between the HLA-DRB1*01 allele and susceptibility to LL and between the HLA-DRB1*08 and protection against leprosy per se in a Mexican Mestizo population, which possesses a high degree of genetic heterogeneity. HLA type studies demonstrated that, in general, Mexican Mestizos harbour several more Amerindian genes compared to European and African haplotypes (29).

In conclusion, our study described the genotyping of the HLA-DRB1 locus in Mexican Mestizos diagnosed with leprosy and healthy individuals. Our findings suggest that the HLA-DRB1*01 and HLA-DRB1*08 alleles may be novel genetic markers for susceptibility to and protection against leprosy, respectively, in a Mexican Mestizo population. The 
investigation of leprosy in different endemic regions may provide more insight into the pathogenesis of this disease and its heterogeneous distribution in Mexico.

\section{Acknowledgements}

The authors thank Dr G. Sanchez-Schmitz (Division of Infectious Diseases, Boston Children's Hospital and Harvard Medical School, Harvard University) for his critical reading. This study was supported by grants from the National Council of Science and Technology-CONACYT (nos. 106152, 79589 and 160883).

\section{References}

1. Gulia A, Fried I and Massone C: New insights in the pathogenesis and genetics of leprosy. F1000 Med Rep 2: 30, 2010.

2. World Health Organization: Global leprosy situation, 2012. Wkly Epidemiol Rec 87: 317-328, 2012.

3. Lavado-Valenzuela R, José Bravo M, Junqueira-Kipnis AP, et al: Distribution of the HLA class II frequency alleles in patients with leprosy from the mid-west of Brazil. Int J Immunogenet 38 255-258, 2011.

4. Alvarado-Navarro A, Montoya-Buelna M, Munoz-Valle JF, Lopez-Roa RI, Guillen-Vargas C and Fafutis-Morris M: The 3'UTR $1188 \mathrm{~A} / \mathrm{C}$ polymorphism in the interleukin-12p40 gene (IL-12B) is associated with lepromatous leprosy in the west of Mexico. Immunol Lett 118: 148-151, 2008.

5. Larrea MR, Carreno MC and Fine PE: Patterns and trends of leprosy in Mexico: 1989-2009. Lepr Rev 83: 184-194, 2012.

6. Vanderborght PR,Pacheco AG, Moraes ME, et al:HLA-DRB1*04 and DRB1*10 are associated with resistance and susceptibility, respectively, in Brazilian and Vietnamese leprosy patients. Genes Immun 8: 320-324, 2007.

7. Misch EA, Berrington WR, Vary JC Jr and Hawn TR: Leprosy and the human genome. Microbiol Mol Biol Rev 74: 589-620, 2010.

8. Alter A, Grant A, Abel L, Alcais A and Schurr E: Leprosy as a genetic disease. Mamm Genome 22: 19-31, 2011.

9. Zhang FR, Huang W, Chen SM, et al: Genomewide association study of leprosy. N Engl J Med 361: 2609-2618, 2009.

10. Miller EN, Jamieson SE, Joberty C, et al: Genome-wide scans for leprosy and tuberculosis susceptibility genes in Brazilians. Genes Immun 5: 63-67, 2004.

11. Hsieh NK, Chu CC, Lee NS, Lee HL and Lin M: Association of HLA-DRB $1 * 0405$ with resistance to multibacillary leprosy in Taiwanese. Hum Immunol 71: 712-716, 2010.

12. Zhang F, Liu H, Chen S, et al: Evidence for an association of HLA-DRB $1 * 15$ and DRB1*09 with leprosy and the impact of DRB $1 * 09$ on disease onset in a Chinese Han population. BMC Med Genet 10: 133, 2009.

13. Ridley DS and Jopling WH: Classification of leprosy according to immunity. A five-group system. Int J Lepr Other Mycobact Dis 34: 255-273, 1966.

14. Rangel-Villalobos H, Munoz-Valle JF, Gonzalez-Martin A, Gorostiza A, Magana MT and Paez-Riberos LA: Genetic admixture, relatedness, and structure patterns among Mexican populations revealed by the Y-chromosome. Am J Phys Antropol 135: 448-461, 2008.
15. Rubi-Castellanos R, Anaya-Palafox M, Mena-Rojas E, Bautista-España D, Munoz-Valle JF and Rangel-Villalobos H: Genetic data of 15 autosomal STRs (Identifiler kit) of three Mexican Mestizo population samples from the States of Jalisco (West), Puebla (Center), and Yucatan (Southeast). Forensic Sci Int Genet 3: e71-e76, 2009.

16. Miller SA, Dykes DD and Polesky HF: A simple salting out procedure for extracting DNA from human nucleated cells. Nucleic Acids Res 16: 1215, 1988.

17. Yamamoto-Furusho JK, Uscanga LF, Vargas-Alarcon G, Rodriguez-Perez JM, Zuniga J and Granados J: Polymorphisms in the promoter region of tumor necrosis factor alpha (TNF-alpha) and the HLA-DRB1 locus in Mexican mestizo patients with ulcerative colitis. Immunol Lett 95: 31-35, 2004.

18. Geluk A and Ottenhoff TH: HLA and leprosy in the pre and postgenomic eras. Hum Immunol 67: 439-445, 2006.

19. Blackwell JM and Searle S: Genetic regulation of macrophage activation: understanding the function of Nramp1 (=Ity/Lsh/Bcg). Immunol Lett 65: 73-80, 1999.

20. Gorodezky C, Flores J, Arevalo N, Castro LE, Silva A and Rodriguez O: Tuberculoid leprosy in Mexicans is associated with HLA-DR3. Lepr Rev 58: 401-406, 1987.

21. Gorodezky C, Alaez C, Munguia A, et al: Molecular mechanisms of MHC linked susceptibility in leprosy: towards the development of synthetic vaccines. Tuberculosis (Edinb) 84: 82-92, 2004

22. Vargas-Alarcón G, Granados J, Rodriguez-Pérez JM, et al: Distribution of HLA class II alleles and haplotypes in Mexican Mestizo population: comparison with other populations. Immunol Invest 39: 268-283, 2010.

23. da Silva SA, Mazini PS, Reis PG, Sell AM, Tsuneto LT, Peixoto PR and Visentainer JE: HLA-DR and HLA-DQ alleles in patients from the south of Brazil: markers for leprosy susceptibility and resistance. BMC Infect Dis 9: 134, 2009.

24. Motta PM, Cech N, Fontan C, et al: Role of HLA-DR and HLA-DQ alleles in multibacillary leprosy and paucibacillary leprosy in the province of Chaco (Argentina). Enferm Infecc Microbiol Clin 25: 627-631, 2007 (In Spanish).

25. Zerva L, Cizman B, Mehra NK, et al: Arginine at positions 13 or 70-71 in pocket 4 of HLA-DRB1 alleles is associated with susceptibility to tuberculoid leprosy. J Exp Med 183: 829-836, 1996.

26. Soebono H, Giphart MJ, Schreuder GM, Klatser PR and de Vries RR: Associations between HLA-DRB1 alleles and leprosy in an Indonesian population. Int J Lepr Other Mycobact Dis 65: 190-196, 1997.

27. Hegazy AA, Abdel-Hamid IA, Ahmed el-SF, Hammad SM and Hawas SA: Leprosy in a high-prevalence Egyptian village: epidemiology and risk factors. Int J Dermatol 41: 681-686, 2002.

28. Rani R, Fernandez-Vina MA, Zaheer SA, Beena KR and Stastny P: Study of HLA class II alleles by PCR oligotyping in leprosy patients from north India. Tissue Antigens 42: 133-137, 1993.

29. Barquera R, Zuñiga J, Hernandez-Diaz R, et al: HLA class I and class II haplotypes in admixed families from several regions of Mexico. Mol Immunol 45: 1171-1178, 2008. 